\title{
Complete amenorrhea following NovaSure endometrial ablation for resistant menorrhagia: a case report
}

\author{
Ibrahim A. Abdelazim ${ }^{1,2}$, Mohannad AbuFaza', Rania H. Farag ${ }^{3}$ \\ ${ }^{1}$ Department of Obstetrics and Gynecology, Ahmadi Hospital, Ahmadi 61008, Kuwait. \\ ${ }^{2}$ Department of Obstetrics and Gynecology, Faculty of Medicine, Ain Shams University, Abbassyia 11566, Cairo, Egypt. \\ ${ }^{3}$ Department of Obstetrics and Gynecology, Dar Al Shifa Hospital, Hawally, Safat 13034, Kuwait.
}

Correspondence to: Dr. Ibrahim A. Abdelazim, Department of Obstetrics and Gynecology, Ahmadi Hospital, Kuwait Oil Company (KOC), P.O. Box 9758, Ahmadi 61008, Kuwait. E-mail: dr.ibrahimanwar@gmail.com

How to cite this article: Abdelazim IA, AbuFaza M, Farag RH. Complete amenorrhea following novasure endometrial ablation for resistant menorrhagia: a case report. Mini-invasive Surg 2018;2:2. http://dx.doi.org/10.20517/2574-1225.2017.30

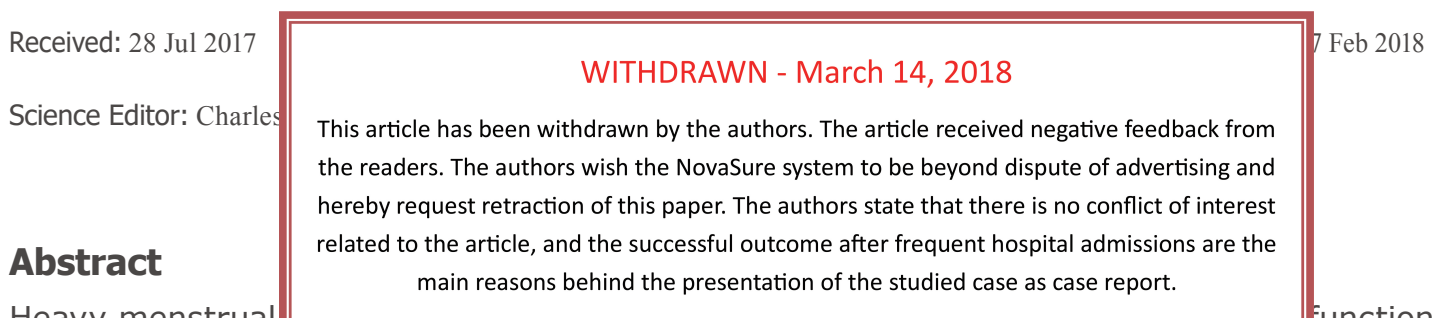

Heavy menstrual

unctional uterine bleeding (DUB). In spite of medical treatment for DUB, many women will eventually require a hysterectomy, which is an invasive treatment option. NovaSure ablation offers a same day noninvasive alternative to hysterectomy and hysteroscopic ablation. A 38-year-old woman presented with HMB in 2015. The attacks of HMB interrupted her lifestyle, and she refused to continue with medical treatment, which failed to resolve her symptoms. This patient was admitted to the hospital four times over 2015 due to the anemia caused by the DUB. Medroxyprogesterone acetate and oral contraceptive pills failed to control the patient's bleeding episodes. Hysteroscopic examination of the uterine cavity showed a normal cavity, and the endometrial biopsy showed proliferative endometrium. She was counseled about NovaSure ablation as the last treatment option before hysterectomy. The NovaSure ablation procedure took $90 \mathrm{~s}$, and the patient was discharged from the hospital $6 \mathrm{~h}$ after the procedure. At follow-up, the patient is completely amenorrheic, and she is satisfied with her results. This study demonstrated that NovaSure endometrial ablation is a safe, effective, non-invasive alternative to hysteroscopic endometrial ablation for treatment of DUB.

Keywords: Amenorrhea, NovaSure, ablation, menorrhagia 


\section{INTRODUCTION}

Heavy menstrual bleeding (HMB) or menorrhagia is the most common form of dysfunctional uterine bleeding (DUB). DUB is the diagnosis of exclusion after ruling out any pelvic pathology and/or medical causes of bleeding ${ }^{[1]}$. HMB or menorrhagia is the most common form of DUB during the reproductive period, it affects $15 \%-20 \%$ of women during their reproductive period. This illness has significant adverse effects on patient quality of the life ${ }^{[2]}$.

In spite of medical treatment for DUB, many women will eventually require a hysterectomy, which is an invasive treatment option ${ }^{[3]}$. Hysteroscopic endometrial ablation, and recently NovaSure endometrial ablation were developed as non-invasive treatment options for DUB after failed medical treatment ${ }^{[4]}$. Compared to hysteroscopic techniques which require proper visualization, and a skilled surgeon, NovaSure ablation technique does not require proper visualization, is less dependent on the surgeon's skills, and eliminates the risk of fluid overload ${ }^{[5]}$.

We present a case report of a relatively young woman who has suffered from DUB for many years and has had frequent hospital admissions and absences from work due to this condition. This woman was successfully treated with NovaSure ablation and she has remained symptom free for more than one and half year after undergoing this proecdeure and has been able to resume her usual daily activities and lifestyle practices.

\section{CASE REPORT}

A 38-year-old wom for one year. She refused to continu had normal thyroi menstrual transva (8-10 cm length),

WITHDRAWN - March 14, 2018

This article has been withdrawn by the authors. The article received negative feedback from the readers. The authors wish the NovaSure system to be beyond dispute of advertising and hereby request retraction of this paper. The authors state that there is no conflict of interest related to the article, and the successful outcome after frequent hospital admissions are the main reasons behind the presentation of the studied case as case report. mptoms persisting and lifestyle. She toms. The patient profiles. Her postd a normal uterus ormal Pap smear, normal hysteroscopic evaluation of the uterine cavity, and the endometrial biopsy taken 3 months prior that showed disordered proliferative endometrium. The patient was admitted to the hospital four times over the course of a year due to anemia caused by the attacks of HMB.

During her first admission, her hemoglobin was $9 \mathrm{gms} / \mathrm{dL}$. She received medical treatment for menorrhagia in of medroxyprogesterone acetate $10 \mathrm{mg}$ daily for 10 days each month for 3 months with tranexamic acid (anti-fibrinolysis) 1-1.5 g 3 times/day, and parenteral iron was used to correct the patient's anemia ${ }^{[6]}$.

In her second admission, her hemoglobin was $9.5 \mathrm{gms} / \mathrm{dL}$. She received medical treatment of oral contraceptive pills twice daily for 7 days, followed by 1 pill/day after reduction of the menstrual flow for the rest of the cycle for 3 months with tranexamic acid, and parenteral iron was used to correct the patient's anemia[6].

During the third admission, the patient received a blood transfusion (hemoglobin was $7 \mathrm{gms} / \mathrm{dL}$ ). She was counseled about diagnostic hysteroscopy under general anesthesia for evaluation of the uterine cavity with an endometrial biopsy, and insertion of a levonorgestrel intra-uterine device (IUD) ${ }^{[6]}$.

The hysteroscopic evaluation showed a normal uterine size, without any gross intrauterine lesions as fibroids or polyps, and the histological examination of the endometrial biopsy taken during hysteroscopy showed disordered proliferative endometrium [Figure 1]. 


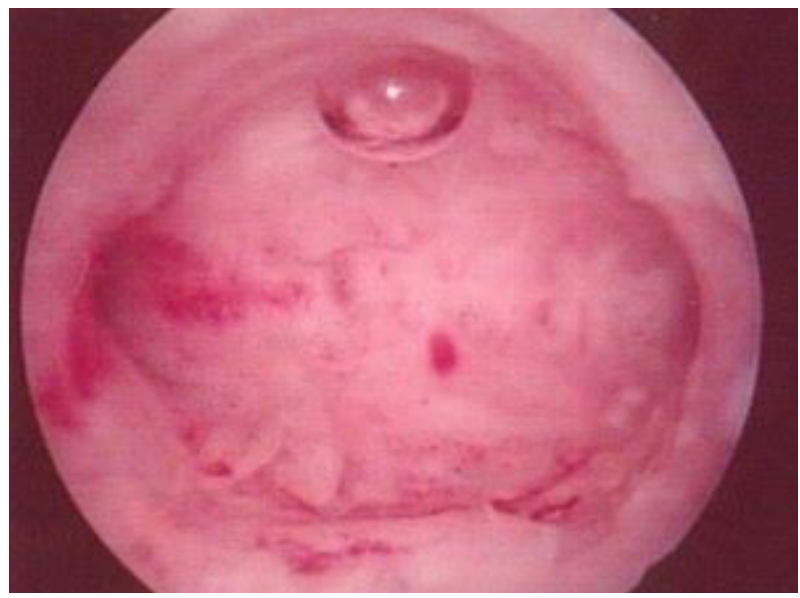

Figure 1: Hysteroscopic examination of the uterus of the patient prior to ablation

In addition, the patient was counseled on the possibility of NovaSure endometrial ablation if the Levonorgestrel IUD failed to control the bleeding episodes, because she had normal size uterine cavity, without intrauterine lesions, and a disordered proliferative endometrium [Figure 1].

The patient was admitted again two months after insertion of the Levonorgestrel IUD due to heavy attack of menorrhagia with expulsion of the Levonorgestrel IUD. During the last admission. she received parenteral iron for anemia. SI risks for future pre injury, perforation, WITHDRAWN - March 14, 2018

This article has been withdrawn by the authors. The article received negative feedback from the readers. The authors wish the NovaSure system to be beyond dispute of advertising and hereby request retraction of this paper. The authors state that there is no conflict of interest In addition, the pa advantage of a no related to the article, and the successful outcome after frequent hospital admissions are the main reasons behind the presentation of the studied case as case report. obtained including rocedure (thermal otting).

lation, such as the and was informed about the hystered

NovSure endometrial ablation was performed immediately after correction of the patient's condition under general anesthesia, and after endometrial curettage, because better outcome obtained if endometrial ablation done with endometrial thickness $<4 \mathrm{~mm}^{[7]}$.

NovaSure system consists of radiofrequency generator [Figure 2], disposable bipolar electrode, suction line desiccant [Figure 3], and a carbon dioxide canister [Figure 4] fixed to the back of the radiofrequency generator ${ }^{[8]}$. First, the carbon dioxide delivered from the radiofrequency generator to the uterus through the central lumen of the disposable bipolar electrode. The disposable bipolar electrode inserted inside the uterine cavity, and when a $50-\mathrm{mmHg}$ intrauterine pressure maintained for $4 \mathrm{~s}$, uterine integrity is confirmed.

Second, while the disposable bipolar electrode was inside the uterine cavity, the radiofrequency generator, measures the uterine cavity width to calculate the total power needed to ablate the endometrium. It then applies negative suction to bring the endometrium in contact with the bipolar electrode before ablation.

Third, the radiofrequency power is generated for ablation of the endometrium and during the ablation technique, the uterine cavity impedance is monitored by the generator to ensure cavity integrity and to avoid uterine perforation ${ }^{[8]}$.

Hysteroscopic examination of the uterine cavity after NovSure endometrial ablation is not mandatory. It was performed in this case in order to counsel the patient about the expected results after the ablation 

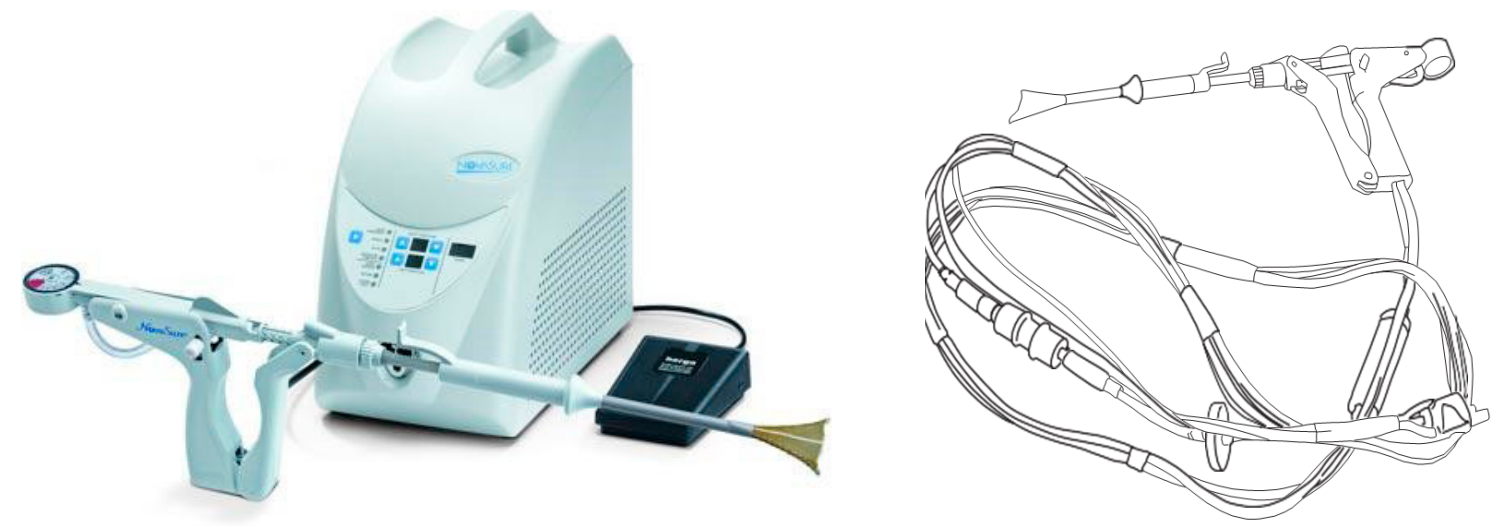

Figure 2: NovaSure system (radiofrequency generator and disposable device with connecting cord, including suction line desiccant)

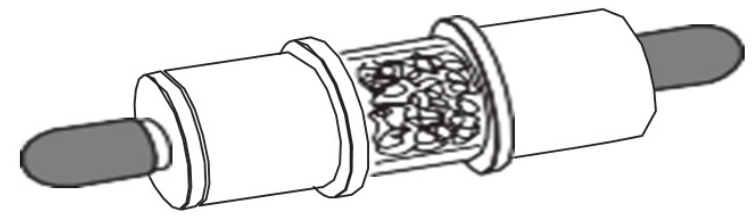

Figure 3: Suction line desiccant

\section{WITHDRAWN - March 14, 2018}

This article has been withdrawn by the authors. The article received negative feedback from the readers. The authors wish the NovaSure system to be beyond dispute of advertising and hereby request retraction of this paper. The authors state that there is no conflict of interest related to the article, and the successful outcome after frequent hospital admissions are the main reasons behind the presentation of the studied case as case report.

procedure. The hysteroscopic examination performed after NovaSure ablation showed complete ablation of the endometrium [Figures 5 and 6; Video 1], and the patient was counseled that she should expect complete amenorrhea after the procedure. The ablation procedure took $90 \mathrm{~s}$ without any intra-operative or postoperative complications. The patient was discharged from the hospital $6 \mathrm{~h}$ after the procedure on nonsteroidal anti-inflammatory (as analgesics).

At follow-up, the patient is completely amenorrheic and she was able to resume her active life style. The patient is completely satisfied with her results and her symptoms have completely resolved.

\section{DISCUSSION}

NovaSure is a second generation non-invasive endometrial ablation procedure that is completed in less than $3 \mathrm{~min}(100-120 \mathrm{~s})$, and was approved by the FDA in $2001^{[8]}$. NovaSure endometrial ablation is indicated for women with DUB after exclusion of endocrine or coagulation disorders, gross uterine cavity pathology (uterine polyps or fibroids), active genital, or pelvic infection ${ }^{[8]}$. Second generation ablation techniques are quick, safe procedures, that do not require fluid media, and are less dependent on the surgeon's skills ${ }^{[9]}$.

Prior systematic reviews of this technique concluded that the second generation ablation techniques were less time consuming compared to the first generation techniques, and can performed under local anesthesia with similar success ${ }^{[3,4]}$. 


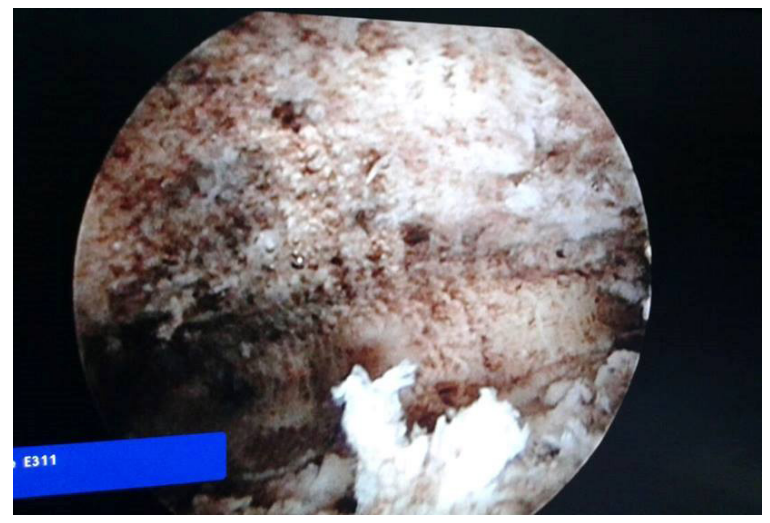

Figure 5: Hysteroscopic examination of the uterus after NovaSure ablation (near view)

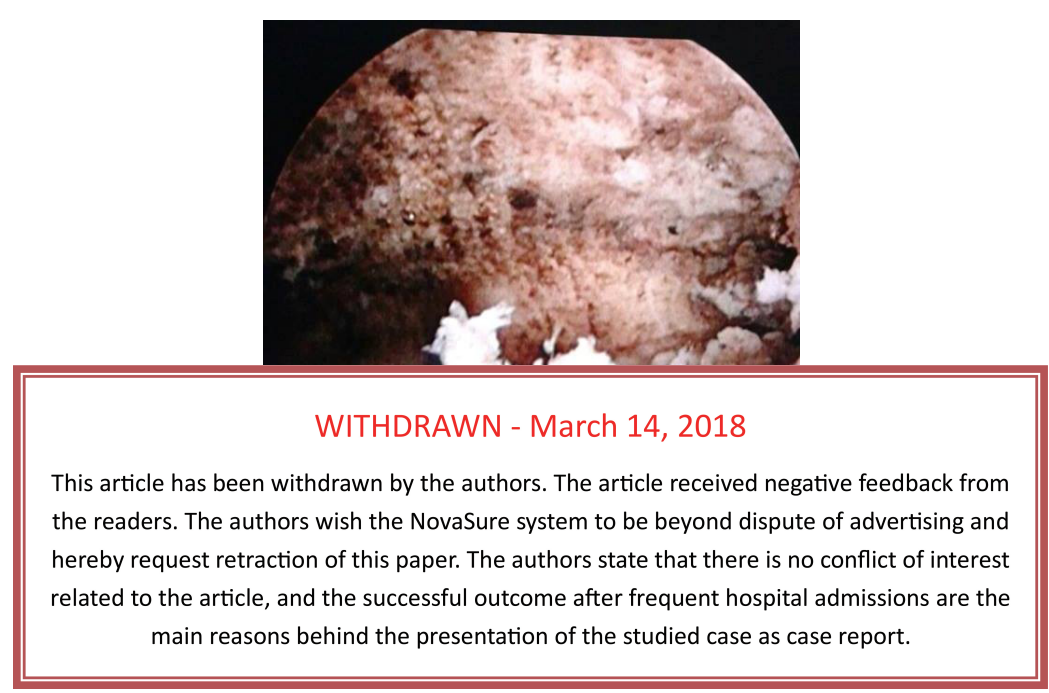

Gallinat ${ }^{[0]}$ concluded that long-term clinical results demonstrate that the NovaSure system is a safe and effective method for treatment of DUB in 107 women studied, with a high success rate, and low re-treatment rates.

Gallinat and Nugen ${ }^{[10]}$ reported that NovaSure is a safe, effective treatment option in DUB after failed medical treatment. In addition; the authors reported $98 \%$ successful reduction in the bleeding attacks with $58.6 \%$ amenorrhea rate at 12 -month follow-up in 107 women with menorrhagia.

Kalkat and Cartmil|[11] studied 50 women with menorrhagia unresponsive to medical management, who were treated by endometrial ablation under local anesthesia. The authors reported a $94 \%(47 / 50)$ improvement in the menstrual bleeding, with $86 \%(43 / 50)$ and $94 \%$ (47/50) satisfaction rate after 4 and 6 months of the NovaSure ablation, respectively.

In addition, the NovaSure ablation technique was evaluated by Thiel et al. ${ }^{[12]}$ in 87 women with uterine length $>10 \mathrm{~cm}$ compared to 101 women with uterine length $\leq 10 \mathrm{~cm}$ (101 women). The authors concluded that the NovaSure endometrial ablation was associated with successful outcomes in women with uterine length $>10 \mathrm{~cm}$, without any serious procedure-related adverse events.

Eighty-one women with HMB were randomly assigned by Clark et al. ${ }^{[13]}$, to bipolar radiofrequency endometrial ablation or thermal balloon ablation in an office setting. Clark et al. ${ }^{[13]}$ reported that the rate of 
amenorrhea was significantly higher in NovaSure-treated women (56\%) compared to ThermaChoice IIItreated women $(23 \%)$ after 12-month follow-up.

In addition, Abbott et al. ${ }^{[14]}$ compared NovaSure endometrial ablation (37 women), and Cavaterm endometrial ablation (18 women) for treatment of DUB. Abbott et al. ${ }^{[14]}$ reported that the rate of amenorrhea was significantly higher in NovaSure-treated women (43\%) compared to Cavaterm-treated women (12\%) after 12-month follow-up.

NovaSure is a safe, non-invasive procedure, with minimal post-operative adverse events including: pelvic cramps, nausea and/or vomiting, hematometra, urinary tract infection. Endometritis after NovaSure ablation is uncommon, and with a report range of $0.6 \%$ to $5 \%$ in clinical studies ${ }^{[14]}$.

In this case report, the patient was admitted to the hospital four times over a one year period due to anemia from bleeding episodes. The anemia was corrected by parenteral iron three times, and a blood transfusion once. The medical treatment (medroxyprogesterone acetate, oral contraceptive pills), and Levonorgestrel IUD failed to control her bleeding epsidoes. NovSure endometrial ablation performed for the patient, followed by hysteroscopic examination of the uterine cavity after NovSure ablation (which is not mandatory). The hysteroscopic examination performed after NovaSure ablation showed complete ablation of the endometrium, and the patient was counseled to anticipate complete amenorrhea after the procedure. At follow up, the patient is completely amenorrheic and she resumed her work and daily activity. She is completely satisfied, after one year of suffering from frequent hospital admissions, and absence from work, because of the he

WITHDRAWN - March 14, 2018
Post-procedure pa the successful out the readers. The authors wish the NovaSure system to be beyond dispute of advertising and of the studied case hereby request retraction of this paper. The authors state that there is no conflict of interest related to the article, and the successful outcome after frequent hospital admissions are the

In conclusion, Nor main reasons behind the presentation of the studied case as case report.

Sure ablation, and d the presentation nent of DUB after failed medical treatment and Levonorgestrel IUD. NovaSure endometrial ablation is a safe, effective, noninvasive alternative to hysteroscopic endometrial ablation. Compared to hysteroscopic ablation techniques, NovaSure ablation does not require proper visualization, is less dependent on the surgeon's skills, and eliminates the risk of fluid overload.

\section{DECLARATIONS}

\section{Acknowledgments}

Authors are grateful to the patient presented in this study for allowing her case to be presented.

\section{Authors' contributions}

Study design, handling the NovaSure ablation, and final revisions before publication: Abdelazim IA Patient's follow up, writing the manuscript, updating the references, and final revision before publication: AbuFaza M Writing the manuscript and revisions before publication: Farag $\mathrm{RH}$

\section{Financial support and sponsorship}

The presentation is funded by the authors themselves.

\section{Conflicts of interest}

There are no conflicts of interest.

\section{Patient consent}

Obtained. 


\section{Ethics approval}

Approved by the departmental meeting of the Obstetrics and Gynecology Department of Ahmadi hospital, Kuwait Oil Company (KOC), Ahmadi, Kuwait.

\section{Copyright}

(C) The Author(s) 2018.

\section{REFERENCES}

1. Abdelazim IA, Abu-Faza M, Hamdan SB. Outcome and hemodynamic alternation in uterine artery following hysteroscopic roller-ball endometrial ablation. J Gynecol Res 2015;1:105.

2. Abdelazim IA, Belal MM. Outcome and uterine arteries Doppler velocimetry after thermal balloon endometrial ablation in menorrhagia. Evid Based Women Health J 2013;3:35-8.

3. Lethaby A, Hickey M, Garry R. Endometrial destruction techniques for heavy menstrual bleeding. Cochrane Database Syst Rev 2009;(4):CD001501.

4. Garside R, Stein K, Wyatt K, Round A, Price A. The effectiveness and cost-effectiveness of microwave and thermal balloon endometrial ablation for heavy menstrual bleeding: a systematic review and economic modelling. Health Technol Assess 2004;8:1-155.

5. Abbott JS, Garry R. The surgical management of menorrhagia. Hum Reprod Update 2002;8:68-78.

6. Wellington K, Wagstaff AJ. Tranexamic acid: a review of its use in the management of menorrhagia. Drugs 2003;63:1417-33.

7. Practice Committee of American Society for Reproductive Medicine. Indications and options for endometrial ablation. Fertil Steril 2008;90:S236-40.

8. Gimpelson RJ. Ten-year literature review of global endometrial ablation with the NovaSure ${ }^{\circ}$ device. Int J Womens Health 2014;6:26980.

9. Gallinat A. NovaSure impedance-controlled system for endometrial ablation: three-year follow-up on 107 patients. Am J Obstet Gynecol 2004;191:1585-9.

10. Gallinat A, Nugent

11. Kalkat RK, Cartmi Gynecol 2011;31:1

12. Thiel JA, Briggs $\mathrm{M}$ length over $10 \mathrm{~cm}$.

13. Clark TJ, Samuels ablation in the offic

14. Abbott J, Hawe J, systems for the tre

\section{WITHDRAWN - March 14, 2018}

This article has been withdrawn by the authors. The article received negative feedback from the readers. The authors wish the NovaSure system to be beyond dispute of advertising and hereby request retraction of this paper. The authors state that there is no conflict of interest related to the article, and the successful outcome after frequent hospital admissions are the main reasons behind the presentation of the studied case as case report. arosc 2002;9:283-9 rvational study. $J$ Obstet men with uterine cavity nal balloon endometrial ure endometrial ablation 